\title{
ANALysis AND Modelling OF POWER CONSUMPTION IN IOT WITH VIDEO QUALITY COMMUNICATION
}

\author{
Tawfeeg S. Noor ${ }^{1}$, Niemah I. Osman ${ }^{1}$ and Is-Haka M. Mkwawa ${ }^{2}$ \\ ${ }^{1}$ College of Computer Science and Information Technology, \\ Sudan University of Science and Technology, Khartoum, Sudan \\ ${ }^{2}$ School of Computing, Electronics and Mathematics, Plymouth University, UK
}

\begin{abstract}
Internet of Things applications such as environmental monitoring and healthcare may involve multimedia communications from IoT devices to humans for decision-making. Therefore, the quality of delivered multimedia should be in good perceived quality. Higher video quality results into higher energy consumptions due to encoding and decoding processes and as a result, will affect the performance of IoT devices due to their inherent energy constraints. This paper presents the impact of video encoding parameters as non-network parameters on the energy consumption of IoT devices. The experimental results from Cooja simulator show that the videos with high bitrates and low frame rates consume more power than videos with low bitrates and high frame rates. It was also found that video content type affects energy consumption. Finally, this paper proposes a power model that takes into account video parameters such as bit rate, frame rate and content types. The proposed model can play a vital role in video quality adaptation in multimedia communication over IoT devices.
\end{abstract}

\section{KEYWORDS}

Internet of things, Delivered multimedia, Energy consumption, Power model

\section{INTRODUCTION}

In recent years video services and the Internet of Things (IoT) have generated growing interest in the computing and networking research community [1]-[8]. Video services is a mature topic that has developed into the most voluminous type of data currently traversing the Internet, with estimated percentage usage above 50\% of all Internet traffic [2], [5], [6]. It is also expected that this ratio will increase, with the increase of the population socially and professionally interacting over the Internet.

Quality of experience is defined as "the overall acceptability of an application or service as perceived subjectively by the end-user " according to ITU-T Rec.p.10G.100 Amendment 2 [9].This definition should include among other factors users' devices, network parameters such as bandwidth and jitter, environment where users are consuming the services or using the application and user expectations [10]-[12] Video quality can be assessed using either subjective or objective method. QoE is normally measured by the Mean Opinion Score (MOS) metric. The MOS metric has a 1 to 5 points scale representing five terms of multimedia quality (bad, poor, fair, good and excellent) [13]. 
The International Journal of Multimedia \& Its Applications (IJMA) Vol.10, No.1/2/3, June 2018

IoT refers not only to mobile phones connected through the Internet but also to the wireless interconnection of billions of "things" and devices through the Internet or local area networks. With these billions of things come billions of batteries that must be purchased, maintained and disposed off.

In the IoT, users can access video services anywhere and anytime using smart devices. These smart devices connect to the Internet via one or more telecommunications operators. Users' expectations along with additional parameters such as cognitive and behavioral states, cost, and network parameters may determine the acceptability of the service from the user's point of view. If users are not satisfied with the service, they may switch to different providers or may stop using a particular application or service. Therefore network and service providers have realized that the traditional parameters to evaluate video service quality such as delay, packet loss and jitter are not enough and therefore, non-network parametrs such as devices power and user context should be taken into considerations.

For IoT devices, memory and power are one of the major constraints. Therefore, in order to deliver acceptable video quality, video encoding parameters should be carefully chosen in order to save devices energy during video transmission without jeopardizing the QoE. Much effort has been put into energy serving techniques based on the transmission protocols. However, a little effort has been put into investigating the impact of non-network parameters such as video encoding parameters on energy consumption over IoT devices and its effect on the video QoE.

The contributions of this paper are as follows,

1) The investigation of the impact of video encoding parameters on the power consumption over IoT devices and its effect on the quality of experience.

2) The development of the power model that takes into account the bit rates, frame rates and the video content type.

3) The use of subjective tests to investigate the quality of the delivered videos over the IoT devices.

The remainder of this paper is structured as follows. Section 2 discusses the IoT background while Section 3 discusses the quality of experience evaluation for IoT. The experimental setup and results are reported in Sections 4 and Section 5, respectively. Our proposed power model is introduced in Section 6. Finally, Section 7 concludes the paper.

\section{IOT BACKGROUND}

The Internet of Things (IoT) is a new paradigm that is increasing in popularity. It is defined as "an interconnection of uniquely identifiable embedded computing things within the existing Internet infrastructure, offering advanced connectivity of things, systems and services that goes beyond machine-to-machine communications and covers a variety of protocols, domains, and applications" [14]. The number of IoT devices will grow exponentially in the coming years, as Cisco estimates that IoT will consist of 50 billion devices connected to the Internet by 2020 [15].

The evolution of hardware has helped in the expansion of IoT, it is cheaper, consumes less power, and nowadays nearly everybody has a mobile phone with the capability to use a whole lot of connection types, so people can interact with objects anywhere anytime. Connecting the devices to each other allows them to share data among themselves and make intelligent decisions based 
on the data. Any physical device which is connected to some sort of network can be called an IoT device.

\subsection{IoT Architecture}

There is no single consensus on architecture for IoT, which is agreed universally. Different architectures have been proposed by different researchers.

The traditional IoT architecture proposed in[16], is based on a layered model which has 3 layers,

1) Physical level layer:

Acquire the physical information of an environment with different scalar devices and then transmit the data to the network layer via the Wireless Sensor Network (WSN) gateway is the main function of this layer.

2) Network layer:

This layer is responsible for connecting to other smart things, network devices, and servers. Its features are also used for transmitting and processing sensor data.

3) Application layer:

This layer is a combination of a service layer and application layer. The service layer stores the information from the network layer and provides decision making, data analysis, and information management. This paper focuses on this layer whereby video encoding parameters are processed. Challenges for using IoT

Here are four major IoT challenges facing IoT solutions:

1) Battery life

Most IoT things, IoT sensors, smart meters, location trackers, and so on use batteries that might need to keep running for months or years. These things are typically small, with space constraints that limit the size of batteries. As a result, preserving battery life becomes critical (the target study of this paper is battery life).

\section{2) Data costs}

The amount of data that will be generated by billions of IoT things will dwarf anything we have seen on the Internet to date. The types of networks becoming prevalent in IoT implementations have stringent limitations on the amount of data that can be sent. Data networks are expensive, and the frequent, bursty messages produced by IoT things can make data costs spiral quickly out of control.

3) Operational efficiency

IoT solutions often communicate over slower wireless networks. This means that more actionable data needs to be extracted faster.

4) Low-power networks

Today's wireless networks, especially the low-power WANs (LPWANs) are increasingly used in the IoT, run at extremely low data rates. These low data rates impose severe limits on the amount 
The International Journal of Multimedia \& Its Applications (IJMA) Vol.10, No.1/2/3, June 2018

of data (especially video transmission and delivery) that can be communicated over a wireless network.

\subsection{RPL: IPv6 Routing Protocol For Low Power and Lossy Networks}

RPL is the IPv6 Routing Protocol for Low-power and Lossy Networks (LLNs), and was designed to be suitable for resource-constrained devices in industrial, home, and urban environments[17]. RPL is the industry standard for IoT WSN. The main goal of RPL is to provide IPv6 connectivity to a large number of battery-operated embedded wireless devices that use low-power radio to communicate and deliver their data over multiple hops. From the initial design phase, RPL builds upon widely-used routing protocols and research prototypes in the Wireless Sensor Network (WSN) domain such as the collection tree protocol (CTP) [18]but is extended and re-designed to be part of, and ready for, IPv6.

LLNs are a class of network in which both the routers and their interconnect are constrained. LLN routers typically operate with constraints on processing power, memory, and energy. RPL provides a mechanism whereby multipoint-to-point traffic from devices inside the LLN towards acentral control point as well as point-to-multipoint traffic from the central control point to the devices inside the LLN is supported. Support for point-to-point traffic is also available.

\section{QoE Evaluation AND PoWer-Driven Video QuALity on IOT}

The emerging categories of IoT objects tend to be mobile, multi-sensorial and smart, such as wearable sensors, Smartphone's, and smart vehicles, bringing also to an increase of multimedia content in the IoT. Multimedia content refers to a combination of two or more different media contents such as text, audio, image and video.

There are a few studies that address the overall QoE over IoT systems. The work in [19] presents a comprehensive model for the power consumption of wireless sensor nodes. The model takes a system-level perspective to account for all energy expenditures: communications, acquisition, and processing. Furthermore, it is based only on parameters that can empirically be quantified once the platform (i.e., technology) and the application (i.e., operating conditions) are defined. This results in a new framework for studying and analyzing the energy life-cycles in applications, and it is suitable for determining in advance the specific weight of application parameters, as well as for understanding the tolerance margins and tradeoffs in the system. In our views, this model needs enhancing and improvement by considering the end user's Quality of Experience (QoE) in the context of video services which is very important when we come to reducing power consumption for IoT devices.

To increase the battery life, the authors in [20] proposed a voice over IP (VoIP) quality adaptation scheme whereby an acceptable quality is maintained by changing video send bitrate in order to conserve power and hence, prolong VoIP communication session. The results have shown the effectiveness of the proposed scheme in terms of power saving while maintaining acceptable QoE. The power saving was between $10-30 \%$ of the total system power. This model was only proposed for mobile device and we can enhance this model by including IoT devices and adding more influential factors such as the frame rate and bitrate to offer an acceptable quality for the end users. 
The work in [21]presents a framework that reduces the energy consumption of wireless mobile devices during streaming video content over Wireless LAN networks by utilizing the power saving mechanisms defined in the IEEE 802.11e standard. The framework allows video applications to directly optimize the overall energy efficiency by controlling the sleep cycles of wireless network adapters based on video QoE. Although the framework was claimed to save 20$30 \%$ of total system power but did not answer some key concerns of practical interests in the real world implementation of VoIP communication. Under the presence of VoIP signaling traffic the sleep cycles technique is not realistic. The ideal technique is to reduce power consumption of the AV application while keeping the VoIP quality at an acceptable QoE level. Changing the frame rates and bitrates for the videos will reduce the power consumption for the IoT devices while offering an acceptable quality for the end users.

In [22] the authors proposed a simple QoE model and several power models relevant to mobile video services which were formulated by considering that user's QoE is constrained by factors like devices and the ambient environment, they also proposed a QoE-driven adaptive streaming scheme to help save radio resources and reduce power consumption. The authors also propose a QoE-driven energy-efficient resource allocation algorithm. When adapting these models the power consumption can be saved at both base station side and user equipment side without decreasing user's QoE. Adding non-networks parameter to these models ensure an acceptable quality of videos services on IoT devices for end users

\section{EXPERIMENTAL SETUP}

In this experiment, three original videos (raw videos) which are Bus, Crew, and Suzie in quarter common intermediate format (QCIF) were used. To verify the impact of the bitrate and frame rate on power consumption for IoT devices, the three videos with video codec $\mathrm{H} 264$ were employed in the experiment with a variety of content as shown in the screenshots of videos (Figure 1). The videos parameters for $\mathrm{H} 264$ are shown in Table 1. The Crew video was categorized as fast movement video because the crew of around 7 people were walking. The Bus video was categorized as medium movement because a bus and a van were in medium motion. The Suzie video sequence was categorized as slow movement because the lady was on the phone and there was a slow movement of her upper body.
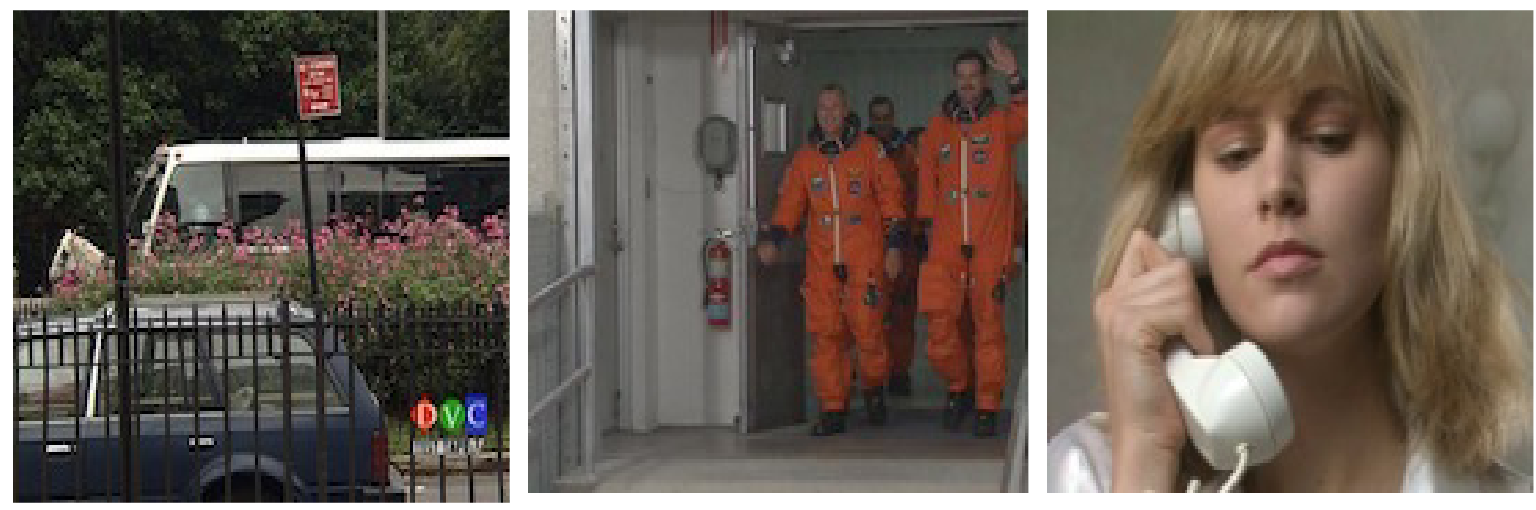

Figure 1: From left, bus, crew and Suzie videos 
The International Journal of Multimedia \& Its Applications (IJMA) Vol.10, No.1/2/3, June 2018

Table 1: videos parameters for $\mathrm{H} 264$

\begin{tabular}{|l|l|}
\hline Video sequence & Bus, Crew, Suzie \\
\hline Codec & H.264 \\
\hline Resolution & $176 \times 144$ \\
\hline Bitrate(kbps) & $30-100$ \\
\hline Framerate & $25,30 \mathrm{fps}$ \\
\hline Encoder & FFmpeg \\
\hline Chroma forma & $4: 2: 0$ \\
\hline
\end{tabular}

To transmit the video sequences the video trace files were generated by using the following steps:

- The mp4 container is used in the experiment to create ISO MP4 files containing the video samples (frames) and a hint track which describes how to packetize the frames for the transport with Real Time Protocol (RTP).

- After creating the hinted mp4 files the mp4 trace tool from EvalVid is able to send a hinted mp4-file per RTP/UDP to a specified destination host.

- When the mp4 trace finishes the transmission of the video, the corresponding video trace files are reconstructed. The relevant data contained in the video trace file is the frame number, the frame type and size and the number of segments in case of (optional) frame segmentation. Table 2 shows the video trace file format.

Table 2: Video trace file format

\begin{tabular}{|l|l|l|l|l|}
\hline $\begin{array}{l}\text { Frame } \\
\text { Number }\end{array}$ & $\begin{array}{l}\text { Frame } \\
\text { Type }\end{array}$ & $\begin{array}{l}\text { Frame } \\
\text { size }\end{array}$ & $\begin{array}{l}\text { Number of UDP- } \\
\text { packets }\end{array}$ & $\begin{array}{l}\text { Sender } \\
\text { Time (ms) }\end{array}$ \\
\hline 1 & I & 180 & 1 & 0.45 \\
\hline 2 & B & 90 & 1 & 0.333 \\
\hline 3 & B & 77 & 1 & 0.2213 \\
\hline
\end{tabular}

Cooja simulator and Instant Contiki 2.7 development environment were used to transmit the video trace file. In this experiment, unicast sender and unicast receiver were used and the power consumption during transmission was recorded. We run the application on one type of wireless sensor network motes EXP430F5438 mote which is equipped with $8 \mathrm{MHz}$ Texas Instruments MSP430 low power microcontroller, $10 \mathrm{~KB}$ RAM and $48 \mathrm{~KB}$ flash[23].

In the subjective test, a total of 29 people took part in the study on a volunteering basis. No participants had any experience working in video quality assessment or coding. There were 13 females and 16 male participants, all of them had normal eye vision. Their ages ranged from 21 to 26 with an average age of 23 . 
The International Journal of Multimedia \& Its Applications (IJMA) Vol.10, No.1/2/3, June 2018

The video in Figure 1 were employed in the experiment where the duration of each video was less than one minute with frame rate ranging from 25-30fps and a resolution of $176 \mathrm{x} 144$. The laptop monitor that was used for display in the test was a 14 inch monitor. All participants were allowed to watch the videos, as many times as they wish before making the decision, but they had to watch them all at least once. Participants evaluated the quality of videos in an uncontrolled environment where they had the freedom of completing the evaluation at home or in their office. Each participant had to randomly (as per ITU-T standard [24] watch 48 videos. They were also asked if they liked the video content

\section{EXPERIMENT RESULTS}

To evaluate the impact of the bitrate and frame rate on power consumption for IoT devices, three videos with different content and different quality were employed.

The P-value results in Table 3 demonstrates that the bitrate parameter has significant $(0.0029<$ 0.05) meaningful addition to the power consumption of the IoT device during video transmission.

Table 3: P-value for bitrates values

\begin{tabular}{llllll}
\hline & df & SS & MS & F & P-value \\
\hline Regression & 1 & 9295.485 & 9295.485 & 24.94 & 0.0016 \\
Residual & 7 & 2608.802 & 372.6859 & & \\
Total & 8 & 11904.29 & & & \\
\hline
\end{tabular}

The P-value in Table 4 illustrates that the frame rate parameter has significant $(0.0016<0.05)$ meaningful addition to the power consumption of the IoT devices Since these p-values are less than the threshold (0.05), frame rate and bitrate parameters are statistically significant to the energy consumption of an IoT device during video transmission over the low power wireless network.

Table 4: P-value for bitrates values

\begin{tabular}{llllll}
\hline & df & SS & MS & F & P-value \\
\hline Regression & 1 & 9295.485 & 9295.485 & 24.94 & 0.0016 \\
Residual & 7 & 2608.802 & 372.6859 & & \\
Total & 8 & 11904.29 & & & \\
\hline
\end{tabular}

According to the results that we obtained from the Cooja simulator as shown in Figure 2, it was observed that the video with higher bitrates and low frame rates consume more power than the videos with lower bitrates and high frame rates. This is because, more data is processed and sent in high bitrates video than in slow and medium video sequences. 
The International Journal of Multimedia \& Its Applications (IJMA) Vol.10, No.1/2/3, June 2018

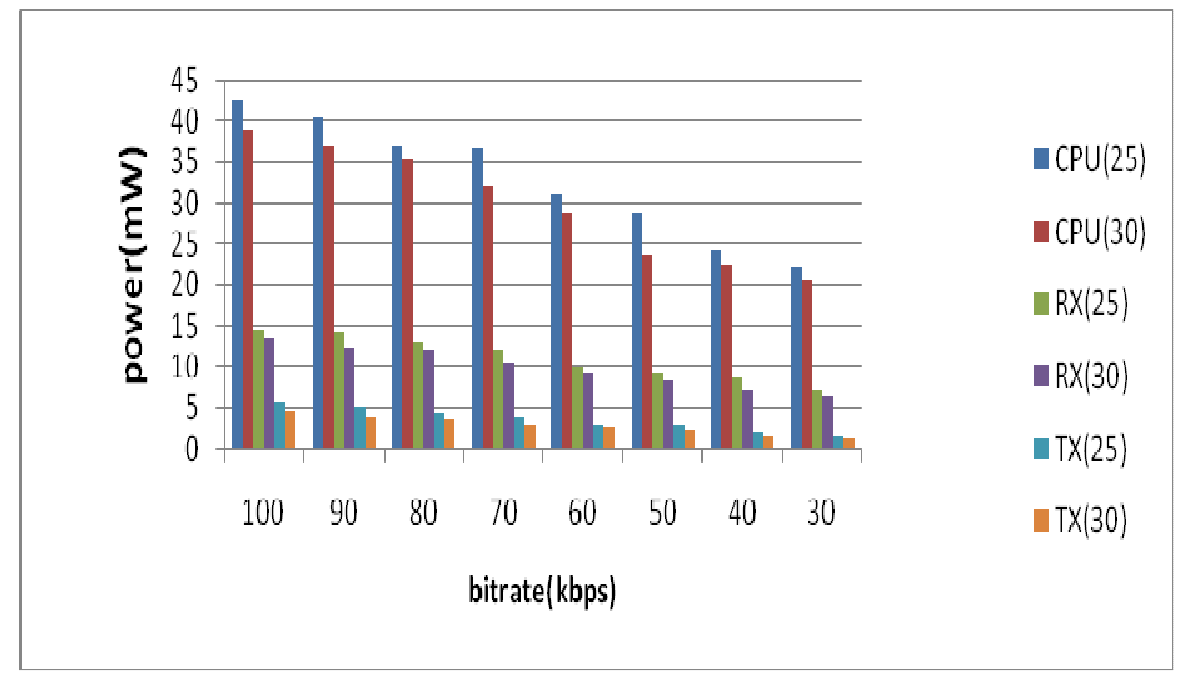

Figure 2. Power consumption of the bus videos

With respect to the bitrate and frame rate parameters, we observed that the videos with fast movement consume more power than the videos with medium and slow movement as shown in Figure 3. This is also due to the fact that, fast movement video sequences are more complex than slow and medium video sequences.

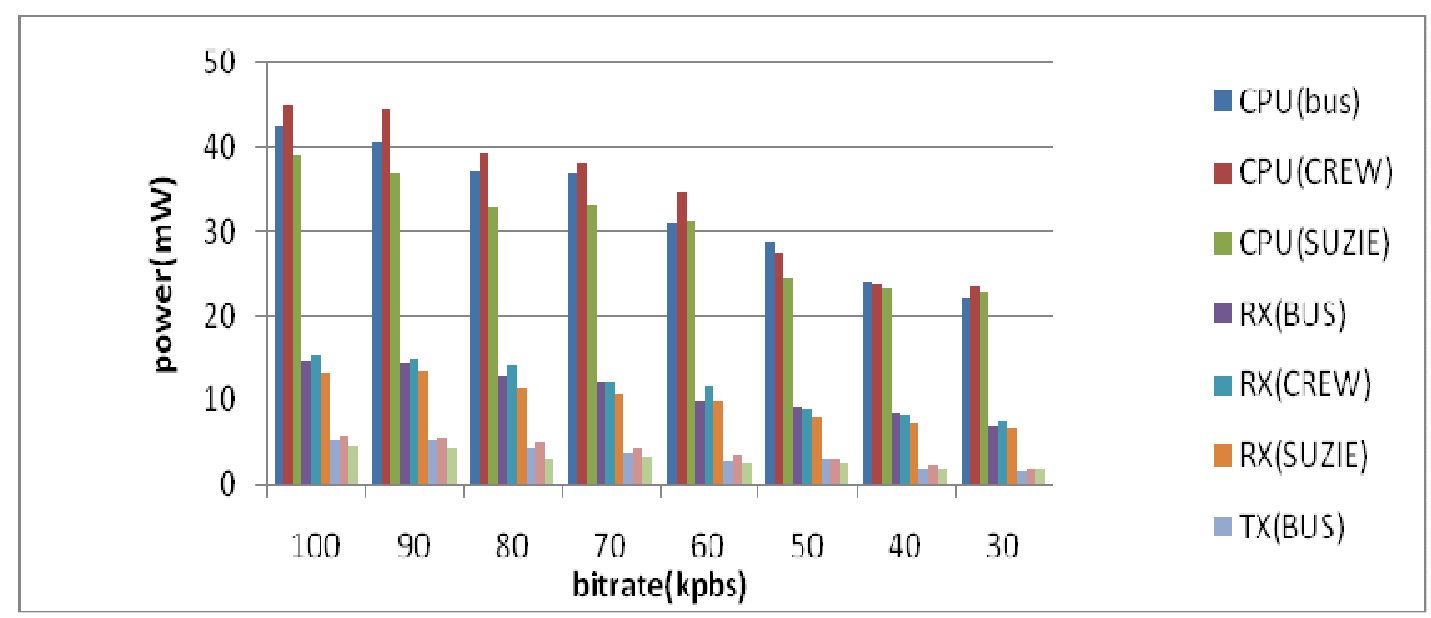

Figure 3. Power consumption for the three videos

To evaluate the power aspects on QoE, three videos with different content and different quality were employed from the reconstructed video sequences transmitted over the IoT devices. There were no packet losses in the entire process. As shown in Figure 4, it was observed that for the same bitrate, frame rate and resolution, slow and medium video sequences recorded better MOS values and hence better quality than fast movement video sequences. This is because fast movement video sequences need high bit rates for better quality compared to slow and medium video sequences. For slow movement video sequence, low frame rates are satisfying for users, because temporal differences are insignificant. 
The International Journal of Multimedia \& Its Applications (IJMA) Vol.10, No.1/2/3, June 2018

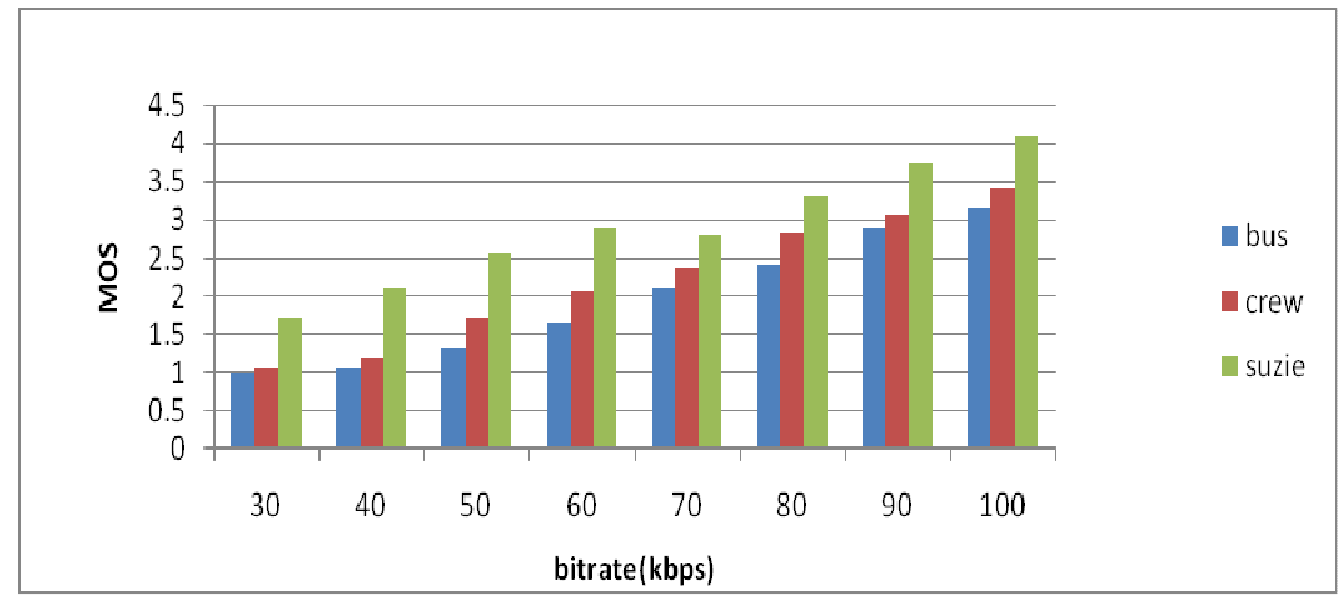

Figure 4 .The Mean Opinion Score

\section{PoWer Consumption MOdeL}

The model for the power consumption is based on the encoding frame rate and bitrate while the encoding resolution was fixed. The smallest QCIF resolution and low bitrates were preferred due to IoT constraints in memory, storage and energy.

Non-linear regression has been used to model the power consumption with the dataset spilt into training (70\% of the data) and testing (30\% of the data). The total power consumption in $\mathrm{mW}$, which is a combination of CPU, data transmission, data receiver and listening cycles, can be expressed as a function of frame rate and bitrate.

$$
\text { Power }=\mathrm{f}(\text { br, fr })
$$

Where, br and fr denotes bitrate and frame rate respectively. From non-linear regression, the total power consumption model is then derived as,

$$
\text { Power }=\alpha * \text { fr } * \ln (b r)+\beta
$$

Where $\alpha$ and $\beta$ are coefficients (Table 5).

The proposed power model in (2) was evaluated by using of $30 \%$ of the dataset for validating the model. The accuracy of the model was determined by using the Root Mean Squared Error (RMSE) and correlation coefficient R2 (Table 6). 
The International Journal of Multimedia \& Its Applications (IJMA) Vol.10, No.1/2/3, June 2018

Table 5: Model coefficients

\begin{tabular}{|l|l|l|l|}
\hline \multirow{2}{*}{ Coefficient } & fast movement & medium movement & slow movement \\
\cline { 2 - 4 } & Crew & Bus & Suzie \\
\hline$\alpha$ & 0.168 & 0.148 & 0.125 \\
\hline$\beta$ & 15.027 & 16.392 & 17.490 \\
\hline
\end{tabular}

Table 6: RMSE and correlation coefficient R2

\begin{tabular}{|l|l|l|l|}
\hline \multirow{2}{*}{$\mathrm{T}$} & fast movement & medium movement & slow movement \\
\cline { 2 - 4 } & Crew & Bus & Suzie \\
\hline R2 & 0.974 & 0.986 & 0.967 \\
\hline RMSE & 2.318 & 16.392 & 1.946 \\
\hline
\end{tabular}

Figure 5-7 depicts the correlation predicted and actual power values of video sequences used in this paper. It can therefore be argued that the proposed power consumption model in IoT with video quality communication can be used by network and service providers as a simple but yet an accurate objective model. The model can be used to control and optimize video quality in IoT devices.

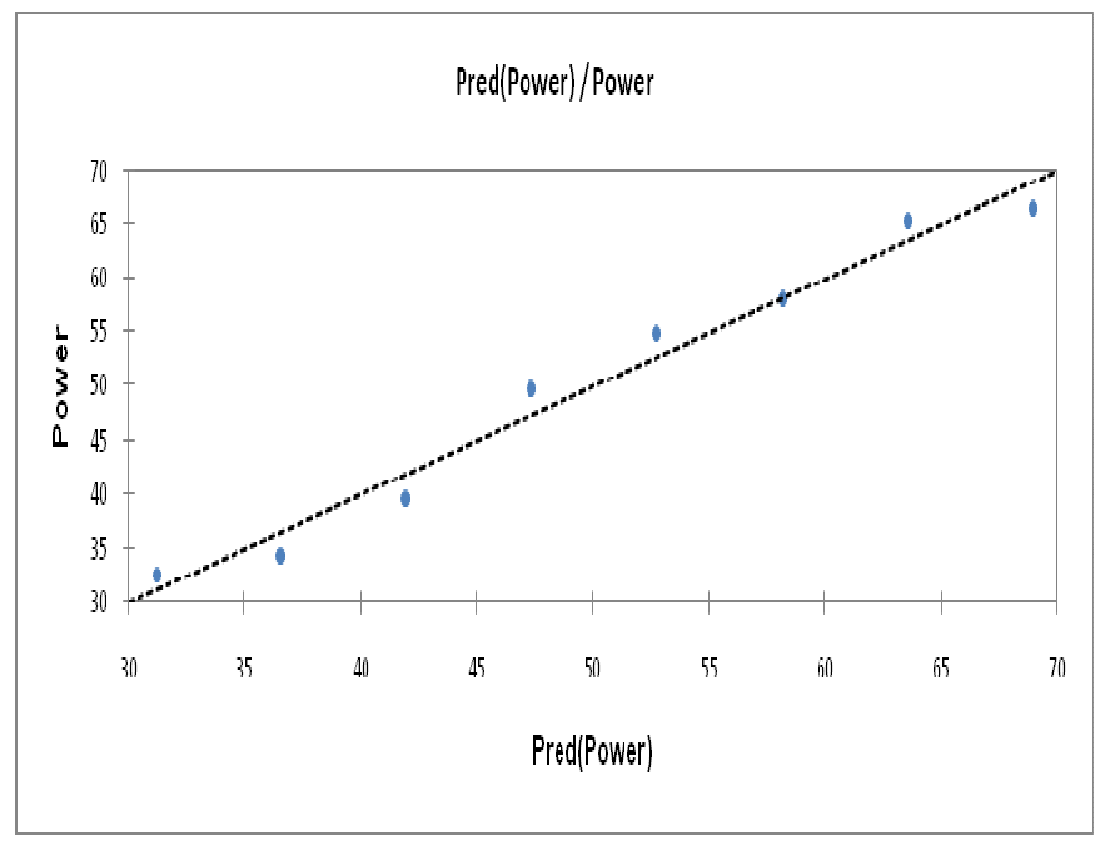

Figure 5. Model validation for fast movement sequences 
The International Journal of Multimedia \& Its Applications (IJMA) Vol.10, No.1/2/3, June 2018

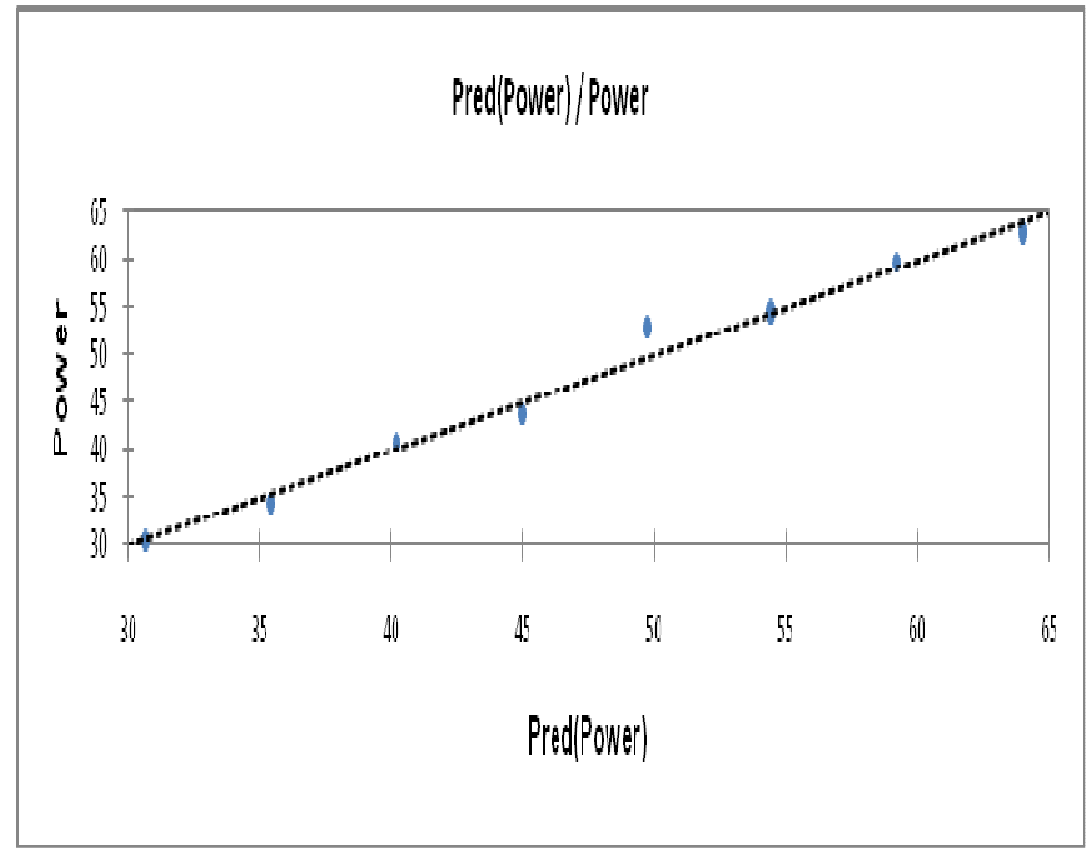

Figure 6. Model validation for medium movement sequences

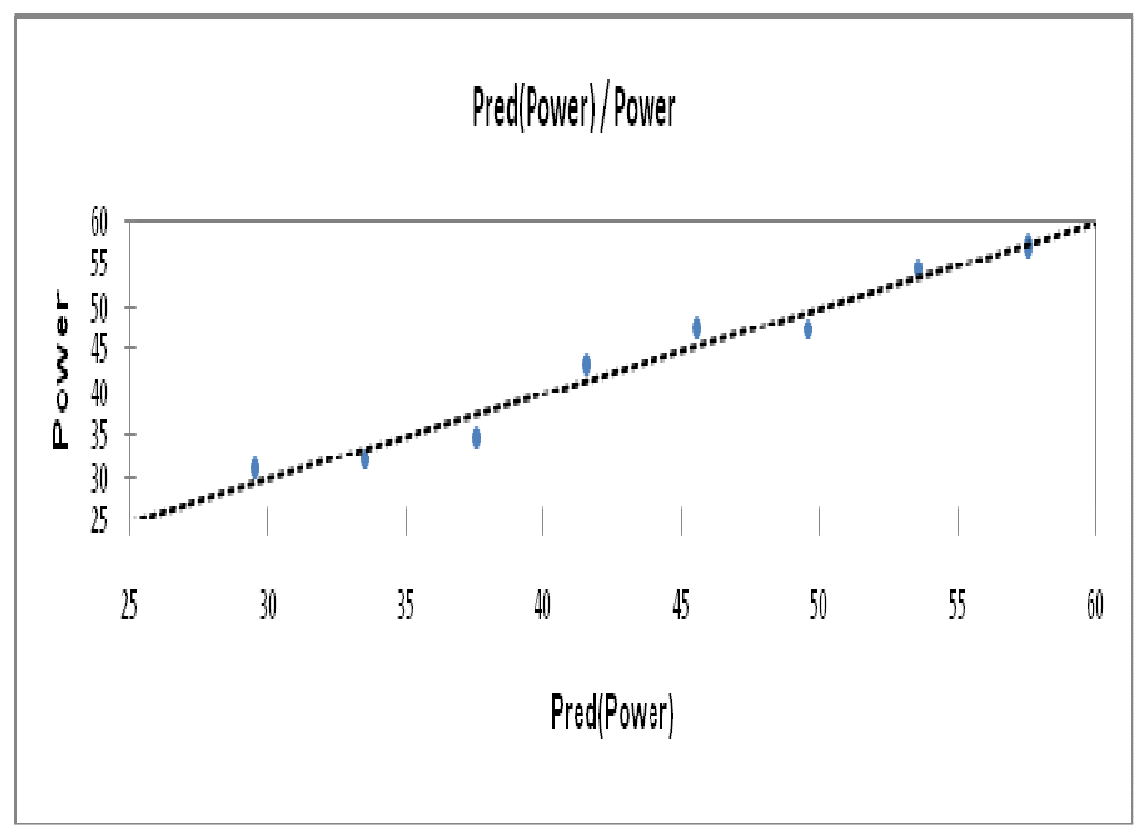

Figureure 7. Model validation for slow movement sequences

\section{Conclusions}

This paper has presented the impact of bitrate and frame rate on the power consumption of IoT devices. It has also proposed the power consumption model, which included bitrates and frame 
The International Journal of Multimedia \& Its Applications (IJMA) Vol.10, No.1/2/3, June 2018

rates as non-network parameters. Experimental results showed that the videos with higher bitrates and low frame rates consumed more power than the videos with low bitrates and high frame rates. It was also found that videos with high movement consume more power than the video with medium and slow movement. The power consumption model can be used to predict power usage of IoT devices. For IoT devices, memory and power are one of the major constraints. Therefore, in order to deliver acceptable video quality, video encoding parameters should be carefully chosen in order to save devices energy during video transmission without jeopardizing the QoE. Future work will include the implementation of the proposed model in IoT systems in order to control and optimize video quality transmission.

\section{REFERENCES}

[1] D. Guinard, V. Trifa, F. Mattern, and E. Wilde, "From the Internet of Things to the Web of Things : Resource Oriented Architecture and Best Practices," Archit. Internet Things, pp. 97-129, 2011.

[2] A. Rao, A. Legout, Y. Lim, D. Towsley, C. Barakat, and W. Dabbous, "Network characteristics of video streaming traffic," Proc. Seventh Conf. Emerg. Netw. Exp. Technol. - Conex. '11, pp. 1-12, 2011.

[3] N. Thomos, S. Argyropoulos, N. V. Boulgouris, and M. G. Strintzis, "Robust transmission of H.264/AVC streams using adaptive group slicing and unequal error protection," EURASIP J. Appl. Signal Processing, vol. 2006, pp. 1-13, 2006.

[4] L. Plissonneau and E. Biersack, "A longitudinal view of HTTP video streaming performance," Proc. 3rd Multimed. Syst. Conf. - MMSys '12, p. 203, 2012.

[5] V. K. Adhikari, S. Jain, Y. Chen, and Z. L. Zhang, "Vivisecting YouTube: An active measurement study," Proc. - IEEE INFOCOM, pp. 2521-2525, 2012.

[6] J. Guo, E. W. M. Wong, S. Chan, P. Taylor, M. Zukerman, and K.-S. Tang, "Performance Analysis of Resource Selection Schemes for a Large Scale Video-on-Demand System,” Multimedia, IEEE Trans., vol. 10, no. 1, pp. 153-159, 2008.

[7] J. Summers, T. Brecht, D. Eager, and B. Wong, "To chunk or not to chunk: Implications for HTTP streaming video server performance," ... Digit. Audio Video, pp. 15-20, 2012.

[8] P. Martinez-Julia, E. T. Garcia, J. O. Murillo, and A. F. Skarmeta, "Evaluating video streaming in network architectures for the internet of things," Proc. - 7th Int. Conf. Innov. Mob. Internet Serv. Ubiquitous Comput. IMIS 2013, pp. 411-415, 2013.

[9] ITU-T P.10/G.100 amd2, "Vocabulary for performance and quality of service," Ser. P Teleph. Transm. Qual. Teleph. Install. Local Line Networks, vol. 100, no. 2006, pp. 1-10, 2008.

[10] M. Robinson et al., "QoE based holistic traffic engineering in SDN enabled heterogeneous transport networks," Int. Conf. Transparent Opt. Networks, pp. 1-4, 2017.

[11] A. Schwind, M. Seufert, Ö. Alay, P. Casas, P. Tran-Gia, and F. Wamser, "Concept and implementation of video QoE measurements in a mobile broadband testbed," TMA 2017 - Proc. 1st Netw. Traffic Meas. Anal. Conf., no. 644399, 2017.

[12]D. Soldani, "Bridging QoE and QoS for Mobile Broadband Networks Curriculum Vitae - Dr . David Soldani," p. 2011, 2011.

[13] ITU-T Recommendation P.910, "Subjective video quality assessment methods for multimedia applications," Int. Telecommun. Union, Geneva, pp. 1-42, 2009.

[14] C. Mulligan and D. Boyle, From Machine-to-Machine to the Internet of Things Introduction to a New Age of Intelligence From Machine-to-Machine to the Internet of Things Introduction to a New Age of Intelligence Stefan Avesand. .

[15] "Internet of Things (IoT)." [Online]. Available: http://www.cisco.com/c/en/us/solutions/internet-of. [Accessed: 25-Jul-2017].

[16] R. Duan, X. Chen, and T. Xing, "A QoS architecture for IOT," Proc. - 2011 IEEE Int. Conf. Internet Things Cyber, Phys. Soc. Comput. iThings/CPSCom 2011, pp. 717-720, 2011. 
The International Journal of Multimedia \& Its Applications (IJMA) Vol.10, No.1/2/3, June 2018

[17] B. A. Klein, "RPL : IPv6 Routing Protocol for Low Power and Lossy Networks,” no. July, pp. 59-66, 2011.

[18] O. Gnawali, R. Fonseca, K. Jamieson, D. Moss, and P. Levis, “Collection Tree Protocol,” 2009.

[19] B. Martinez, M. Montón, I. Vilajosana, and J. D. Prades, "The Power of Models: Modeling Power Consumption for IoT Devices,” IEEE Sens. J., vol. 15, no. 10, pp. 5777-5789, 2015.

[20] I. H. Mkwawa and L. Sun, "Power-driven VoIP quality adaptation over WLAN in mobile devices," 2012 IEEE Globecom Work. GC Wkshps 2012, pp. 1276-1281, 2012.

[21] M. Csernai and A. Gulyás, "Wireless adapter sleep scheduling based on video QoE: How to improve battery life when watching streaming video?," Proc. - Int. Conf. Comput. Commun. Networks, ICCCN, 2011.

[22] B. H. Chunlei Xie , Xin Zhang, Yijie Li, “QoE-driven energy efficiency promotion for mobile video service," pp. 1030-1035, 2015.

[23]E. Board, “MSP-EXP430F5438 Experimenter Board User â€TM s Guide,” no. June, 2011.

[24] O. A. Methods, "ITU-T," 2016.

\section{AUTHORS}

Tawfeeg S. Noor received the BSc. in Computer Science in 2009 from the University of Gezira in Gezira, Sudan and the MSc. in Computer science in 2012 from the Osmania University in Hyderabad, India . He is currently pursuing his PhD degree in Computer Science at Sudan University of Science and Technology in Khartoum, Sudan. His research interests include Quality of Experience in Multimedia Communication, Computer Networking, power saving in IoT and Wireless Sensor Networks.

Niemah Izzeldin Osman received the B.Sc. degree (first class honours) in Computer Science from Sudan University of Science and Technology, Khartoum, Sudan, in 2002 and the M.Sc. degree (with distinction) in Mobile Computing from the University of Bradford, U.K., in 2006 and the Ph.D. degree in Communication Networks from the University of Leeds, U.K in 2015. She is currently an Assistant Professor at the Department of Computer Systems and Networks, Sudan University of Science and Technology, Sudan. Her current research interests include performance evaluation of 4G LTE networks, Internet of Things and QoE of video services.

Is-Haka Mkwawa received his $\mathrm{PhD}$ in computing from the University of Bradford, UK, in 2004. He is currently with the school of computing, electronics and mathematics at the University of Plymouth. His research interests include QoE control and management, mobility management in mobile and wireless networks, Software Defined Networking, power saving in IoT, overlay networks, performance analysis and evaluation of IMS mobility management, parallel computing, IMS media plane security for next generation of emergency communication and services and collective communication. 